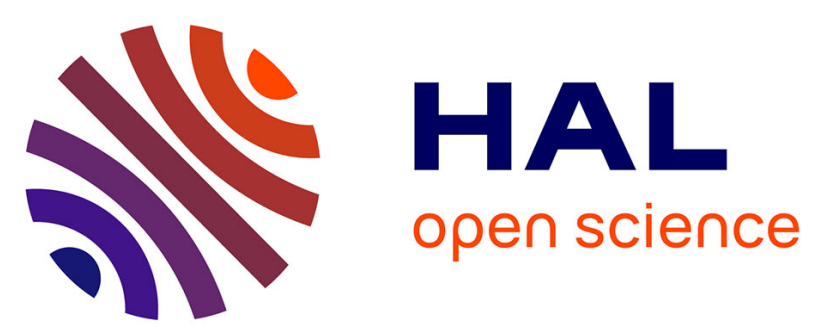

\title{
Application d'un lit fluidisé à la réalisation d'une machine de fluage pour la mesure des grands allongements
}

\author{
C. Oytana, P. Delobelle, D. Varchon
}

\section{To cite this version:}

C. Oytana, P. Delobelle, D. Varchon. Application d'un lit fluidisé à la réalisation d'une machine de fluage pour la mesure des grands allongements. Revue de Physique Appliquée, 1976, 11 (1), pp.159161. 10.1051/rphysap:01976001101015900 . jpa-00243959

HAL Id: jpa-00243959

https://hal.science/jpa-00243959

Submitted on 1 Jan 1976

HAL is a multi-disciplinary open access archive for the deposit and dissemination of scientific research documents, whether they are published or not. The documents may come from teaching and research institutions in France or abroad, or from public or private research centers.
L'archive ouverte pluridisciplinaire HAL, est destinée au dépôt et à la diffusion de documents scientifiques de niveau recherche, publiés ou non, émanant des établissements d'enseignement et de recherche français ou étrangers, des laboratoires publics ou privés. 
Classification

Physics Abstracts

$0.605-7.200-9.100$

\title{
APPLICATION D'UN LIT FLUIDISÉ A LA RÉAlisATION D'UNE MACHINE DE FLUAGE POUR LA MESURE DES GRANDS ALLONGEMENTS
}

\author{
C. OYTANA, P. DELOBELLE et D. VARCHON
}

Laboratoire de Mécanique Appliquée associé au C. N. R. S. Faculté des Sciences, 25030 Besançon Cedex, France

(Reçu le 10 mars 1975, révisé le 16 juin 1975, accepté le 31 juillet 1975)

\begin{abstract}
Résumé. - Description d'une machine de fluage à contrainte constante et à grands allongements utilisant un lit fluidisé comme élément de chauffage. Le lit fluidisé permet l'obtention d'une excellente stabilité en température et d'un gradient thermique presque nul ; propriétés particulièrement intéressantes pour éviter des inhomogénéités dans la déformation.
\end{abstract}

\begin{abstract}
We describe a constant stress creep machine allowing large elongation which uses a fluidised bath as a heating element. The use of a fluidised bath gives good temperature stability and negligible thermal gradient; these properties are particularly useful to avoid strain-inhomogeneities.
\end{abstract}

1. Intérêt de l'appareillage. - L'étude du fluage à chaud à température supérieure à l'ambiante des matériaux présentant de grands allongements

$$
\left(\frac{\Delta l}{l_{0}}>200 \%\right)
$$

soulève un certain nombre de difficultés.

Il est nécessaire d'avoir une longueur initiale d'éprouvette suffisante $(\sim 25 \mathrm{~mm})$ pour éliminer l'influence des points d'ancrage. Il devient donc indispensable de disposer d'un four dont la zone homogène est grande malgré une longueur totale de four réduite afin de permettre la mise en place de la machine de fluage.

$\mathrm{Au}$ cours de l'essai, la modification des conditions aux limites due au mouvement du mors mobile entraîne la formation d'un gradient dynamique. On atteint couramment des écarts de $4^{\circ} \mathrm{C}$ pour des allongements de l'ordre de $250 \%$ au voisinage de $500{ }^{\circ} \mathrm{C}$.

L'existence d'un gradient thermique perturbe l'écoulement du matériau et favorise l'apparition d'une striction [1].

Le mouvement du mors mobile peut également entraîner une instabilité de la température dans le temps préjudiciable à l'essai à cause de l'activation thermique.

La compensation des fours à enroulements devient rapidement délicate dans le cas des grands allongements. Il est donc préférable d'utiliser un bain thermostatique. Les huiles habituellement employées dans ces installations ne permettent guère de dépasser $300^{\circ} \mathrm{C}$, les bains de sel sont d'un emploi peu commode présentant l'inconvénient de se solidifier et de ne pou- voir convenir que dans une plage réduite de température, les lits fluidisés peuvent, par contre, permettre de disposer d'un bain susceptible de supporter une plage de température très étendue avec une inertie thermique importante.

Le lit fluidisé (modèle Heurtey) utilisé est constitué de corindon à grains très fins dans lequel est insufflé de l'air qui le fluidise. On obtient alors l'équivalent d'un liquide brassé. La répartition et l'homogénéisation du gaz sont obtenues en le faisant passer à travers une grille finement percée et un lit fixe constitué de corindon grossier. Le lit fluidisé placé dans un moufle est chauffé par résistances massives. Il est régulé par thyristors avec un oscillant de température de $\pm 0,5^{\circ} \mathrm{C}$ en régime permanent. Il permet de travailler entre l'ambiante et $1100^{\circ} \mathrm{C}$.

2. Réalisation. - La machine de fluage est du type vertical. Les principales difficultés rencontrées dans la mise en place d'une machine de fluage dans un lit fluidisé proviennent de l'incompatibilité entre la présence de sable et les divers mécanismes mis en œuvre. La figure 1 montre un plan d'ensemble de la machine. Elle est constituée de quatre platines (21) (7) (8) (13) reliées par six colonnes (1) (15) en acier inoxydable. Cela conduit à deux sous-ensembles séparés par les platines (7) et (8).

Le premier entre (8) et (13) est immergé dans le lit pendant l'essai. Il comprend le mors fixe (12) et la partie inférieure (10) (constituée par un tube d'alumine frittée) du mors mobile. Le tube amovible (11) et le 


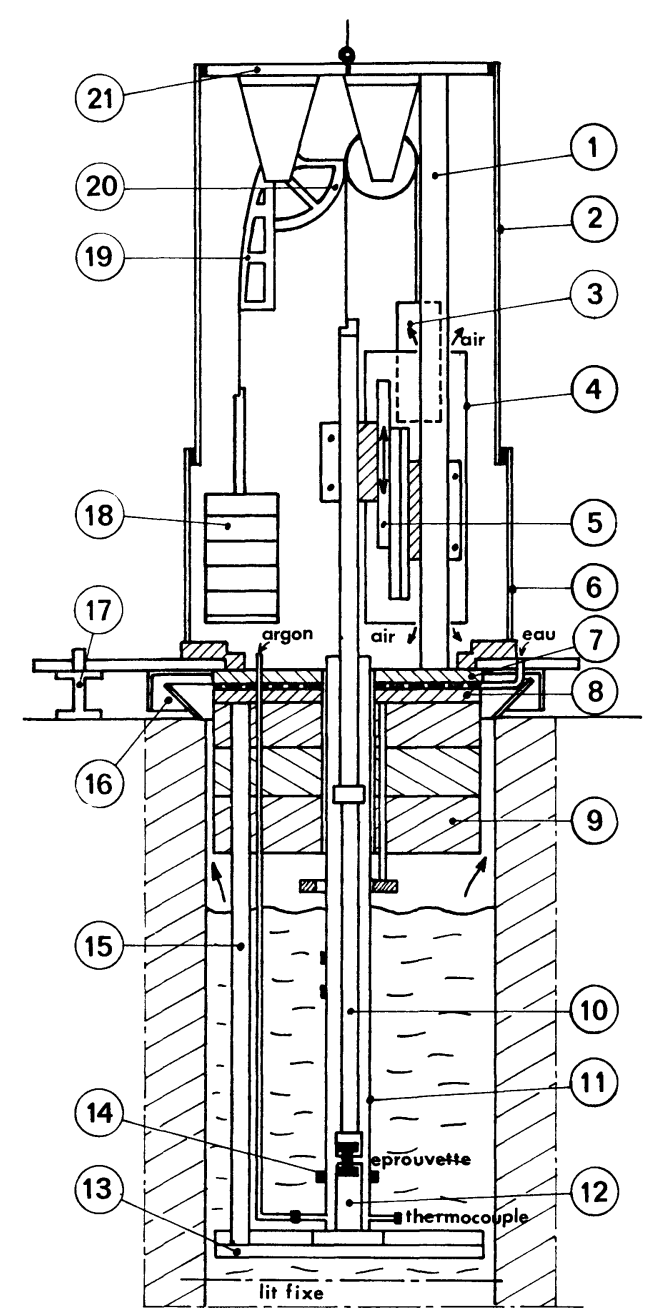

Fig. 1. - Ensemble machine de fluage, lit fluidisé.

double piège à sable (14) permettent de protéger l'éprouvette et les mors tout en permettant la mise en place des éprouvettes.
La partie supérieure entre (7) et (21) est protégée du flux thermique d'une part par les écrans (9) en diatisol et d'autre part par une circulation d'eau entre les platines (7) et (8). Elle comporte les différents éléments de guidage, de mise en charge et de mesure.

Le guidage du mors mobile est assuré le long d'une colonne par une glissière sur rail (5) de $205 \mathrm{~mm}$ de course et protégée du sable résiduel par un capot fermé (4) dans lequel règne une légère surpression d'air. L'alignement des deux mors est réglé au montage et si un léger défaut apparaît il est corrigé par la forme hémisphérique des embouts vissés sur l'éprouvette. La masse du mors mobile est compensée par un contrepoids (3). Les forces de frottement sont inférieures à cent grammes.

La mise en charge de l'éprouvette est assurée par les masses (18), le secteur circulaire (20), la came (19) dont la fonction est de maintenir la contrainte constante au cours de l'allongement (étant admis que le volume reste constant et que la déformation est homogène). Les liaisons entre ces éléments sont assurées par du feuillard de $0,1 \mathrm{~mm}$ d'épaisseur en acier Hasberg. Pour faciliter la mise en place des poids et permettre un départ instantané de l'essai la glissière est commandée par un frein électromagnétique non représenté sur la figure. Celui-ci est en outre utilisé comme sécurité en arrêtant le mors mobile soit en fin de course, soit lorsque après rupture de l'éprouvette sa vitesse dépasse une valeur seuil prédéterminée, ce à l'aide d'un système électronique commandé par le potentiomètre de mesure (Fig. 2).

On mesure le déplacement au niveau de la glissière mobile (5). Les déformations élastiques de la machine sont négligeables par rapport à celles de l'éprouvette. Par contre, la dilatation thermique ne l'est pas, on ne doit donc commencer l'essai que lorsque la machine est stable en température (10 $\mathrm{min})$. Les mesures de dépla-

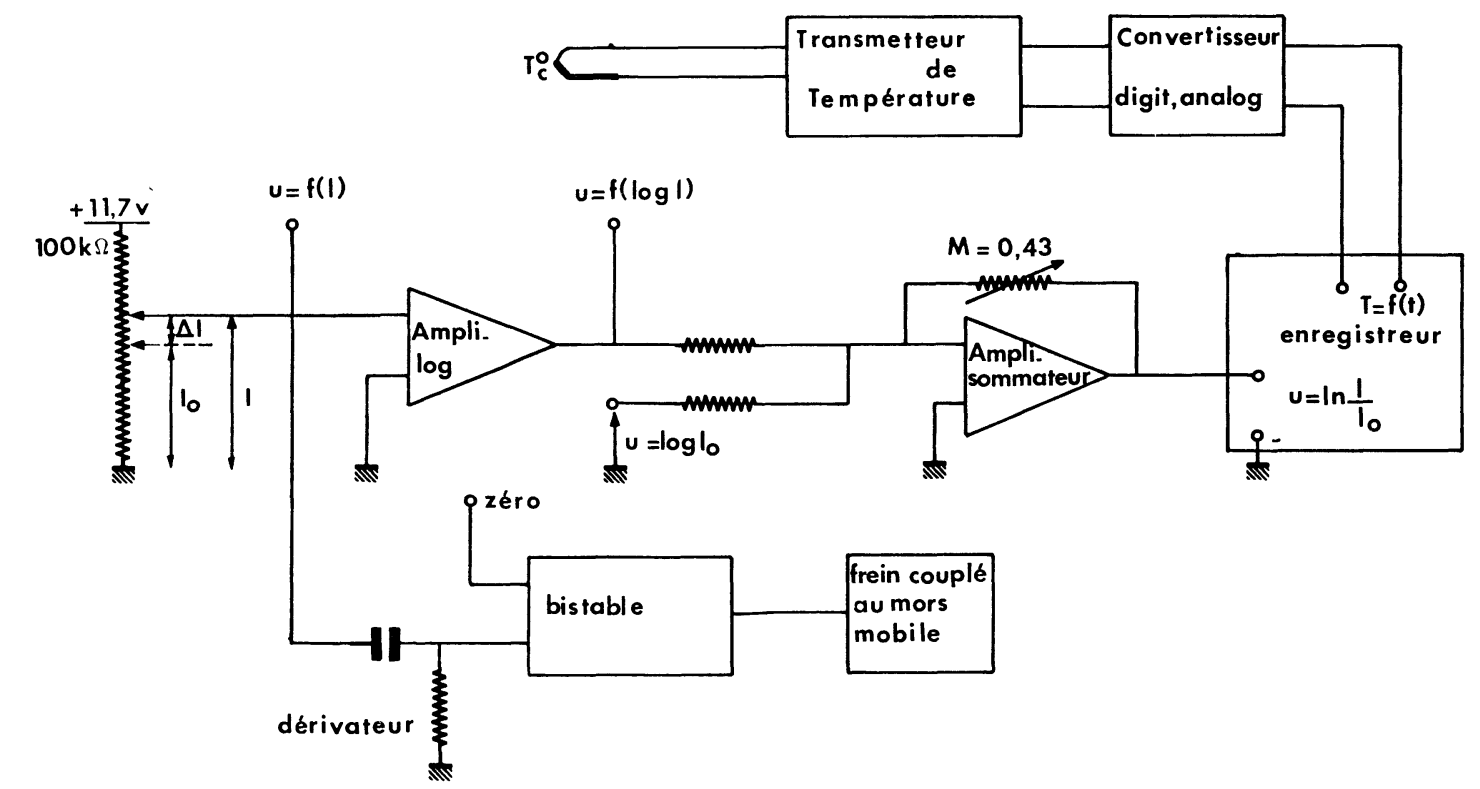

Fig. 2. - Schéma de principe de la mesure de la déformation rationnelle, de la température et de la commande du frein. 
cement peuvent s'effectuer de deux façons : par transformateur différentiel pour les faibles allongements $\leqslant 10 \mathrm{~mm}$ ou par potentiomètre de précision au-delà. Pour enregistrer directement la déformation rationnelle $\bar{\varepsilon}=\operatorname{Ln} \frac{l}{l_{0}}$ en fonction du temps nous réalisons le montage suivant. La tension délivrée par le potentiomètre proportionnelle à la longueur instantanée de l'éprouvette $l$ attaque un amplificateur logarithmique qui délivre une tension $V=k \log l$ à sa sortie. On lui soustrait ensuite une tension constante $k \log l_{0}$. Le résultat est amplifié de nouveau et délivre sur un enregistreur une tension proportionnelle à $\operatorname{Ln} \frac{l}{l_{0}}$ (Fig. 2). La mesure de la température de l'éprouvette est assurée par un thermocouple chromel alumel placé près du mors fixe.

La machine est positionnée à la partie supérieure du lit par trois gougeons de centrage (17). Afin d'éviter des projections trop importantes de corindon des chicanes (16) ont été réalisées. De plus l'ensemble supérieur est protégé des poussières par une vitrine amovible en plexiglass (2) (6). Une circulation d'eau froide dans les trois colonnes (1) assure une température intérieure inférieure à $25^{\circ} \mathrm{C}$ compatible avec le bon fonctionnement des capteurs.

Il est possible de réaliser des essais de fluage en atmosphère inerte.

Pour mettre l'éprouvette en place un treuil permet de soulever l'ensemble de la machine hors du lit fluidisé.

3. Performances. - Nous avons testé cet appareillage à l'aide de résultats obtenus sur des matériaux partiellement étudiés sur une autre machine [2] (laiton $\beta, \mathrm{AlZn}$, et $\mathrm{AlSi}$ ).
La montée en température dure 30 à 35 min. entre deux essais, le lit accusant une baisse de température d'environ $15^{\circ} \mathrm{C}$ après la mise en place de l'éprouvette. Dans le cas d'essais de très courte durée (quelques minutes) ce temps peut être diminué $(10 \mathrm{~min}$.) en démarrant l'essai avant l'équilibre définitif.

Le gradient thermique entre les mors est inférieur à $0,5^{\circ} \mathrm{C}$ sur environ $400 \mathrm{~mm}$ lorsque le mors mobile se déplace. La stabilité de température est de $\pm 0,5^{\circ} \mathrm{C}$ dans le cas le plus défavorable (essai de fluage d'une durée supérieure à une semaine).

L'accord entre les vitesses de fluages obtenues sur les différentes machines est excellent pour les trois alliages. On peut mesurer des vitesses rationnelles allant de $10^{-9} \mathrm{~s}^{-1}$ à $10^{0} \mathrm{~s}^{-1}$.

L'amélioration obtenue par l'utilisation du lit fluidisé est particulièrement frappante dans le cas de l'alliage superplastique $\mathrm{Al}-\mathrm{Zn}$. Alors que l'on observe sur une machine à enroulements compensés plusieurs strictions à $600 \%$ d'allongements, la déformation reste homogène dans le lit fluidisé (l'irrégularité sur le diamètre est inférieure à un dixième de $\mathrm{mm}$ le long d'une éprouvette d'un diamètre final de $1 \mathrm{~mm}$ ).

La charge minimale applicable au mors mobile est de l'ordre de $1 \mathrm{~kg}$ (afin de rendre les frottements négligeables). En l'état actuel de la machine la charge maximum est de $100 \mathrm{~kg}$.

Cette machine est actuellement en fonctionnement permanent et montre que l'utilisation d'un bain thermostatique à lit fluidisé est particulièrement bien adapté aux essais de fluage des matériaux à grands allongements.

Remerciements. - Nous tenons à remercier MM. Laithier et Myotte, Techniciens C. N. R. S. pour leur participation à la réalisation et à la mise au point de cet appareillage.

\section{Bibliographie}

[1] Hart, E. W., "Theory of the tensile test ", Acta Metall. 15 (1967) 351-355.

[2] Mermet-Grandfille, A., «Contribution à l'étude des propriétés mécaniques du laiton $\beta$ aux températures élevées ", thèse $3^{e}$ cycle, Besançon (1973). 